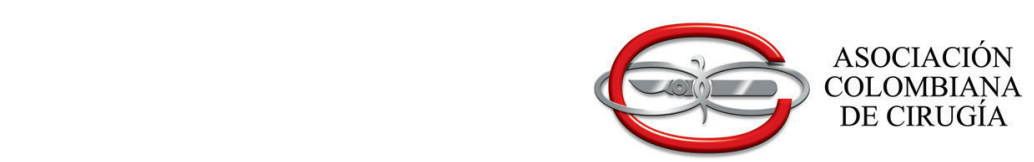

\title{
Recomendaciones para cirugía en pacientes con patologías oncológicas durante la pandemia COVID-19
}

\author{
Recommendations for surgery in patients with oncological pathologies \\ during the COVID-19 pandemic
}

Maikel A. Pacheco ${ }^{1}$, Pilar Adriana Torres², Fernando Arias ${ }^{3}$, Raúl Pinilla ${ }^{4}$, Mario Abadía 5 , Ricardo Villarreal ${ }^{6}$, Alden Gómez ${ }^{7}$, Giovanni Bonilla ${ }^{8}$, Gilbert F. Mateus L. ${ }^{9}$, Lilian Torregrosa ${ }^{10}$, Francisco Henao ${ }^{11}$, Óscar Guevara ${ }^{12}$, Alejandro Múnera ${ }^{13}$, Juan Pablo Villate ${ }^{14}$, Nelson Niño ${ }^{15}$, Carlos Millán ${ }^{16}$

1 Médico, Especialista en Cirugía General, Cirugía Oncológica y Cirugía de Cabeza y Cuello. Profesor asistente y coordinador académico de Cirugía General, Fundación Universitaria de Ciencias de la Salud. Jefe de Servicio, Hospital Infantil Universitario de San José. Cirujano Oncólogo, Hospital de San José, Bogotá, D.C., Colombia.

2 Médica, Especialista en Cirugía General, Oncología Peritoneal, Cirugía Gastrointestinal y Endoscopia Digestiva, Hospital Regional de Sogamoso y Centro Médico Especializado Gastrolife SAS, Duitama, Colombia.

3 Médico, Especialista en Cirugía General, Cirugía Mínimamente Invasora, Jefe Cirugía de citoreducción y quimioterapia hipertérmica intraperitoneal, Fundación Santa Fe de Bogotá. Bogotá DC, Colombia.

4 Médico, Especialista en Cirugía General, Cirugía Gastrointestinal y Endoscopia Digestiva, Instituto Nacional de Cancerología, Universidad Militar Nueva Granada. Bogotá DC, Colombia.

5 Médico, Especialista en Cirugía General, Cirugía Gastrointestinal y Endoscopia Digestiva, Instituto Nacional de Cancerología. Bogotá DC, Colombia.

6 Médico, Especialista en Cirugía General, Cirugía Gastrointestinal y Endoscopia Digestiva, Universidad El Bosque. Bogotá DC, Colombia.

7 Médico, Especialista en Cirugía General, Universidad del Valle, Hospital Universitario del Valle. Cali, Colombia

8 Médico, Especialista en Cirugía General y Cirugía oncológica, Centro de Investigaciones Oncológicas, Compensar EPS. Bogotá DC, Colombia.

9 Médico, Especialista en Cirugía General y Cirugía oncológica, Clínica Amiga. Profesor, Cirugía general, Pontificia Universidad Javeriana. Cali, Colombia.

10 Médico, Especialista en Cirugía General y Cirugía de mama y tumores de tejidos blandos, Hospital Universitario San Ignacio. Bogotá DC, Colombia. Directora departamento de Cirugía, Hospital Universitario San Ignacio, Bogotá; Presidente, Asociación Colombiana de Cirugía.

11 Médico, Especialista en Cirugía General, Hospital Universitario San Ignacio, Pontificia Universidad Javeriana. Bogotá DC, Colombia. Ex presidente, Asociación Colombiana de Cirugía.

12 Médico, Especialista en Cirugía General, Cirujano Hepato-Pancreato-Biliar, Instituto Nacional de Cancerología. Profesor titular, Departamento de Cirugía, Universidad Nacional de Colombia. Bogotá DC, Colombia.

13 Especialista en Cirugía General y Cirugía oncológica, Instituto Nacional de Cancerología. Bogotá DC, Colombia.

14 Médico, Especialista en Cirugía General y Cirugía de Tórax, Fundación Universitaria Ciencias de la Salud. Profesor, Universidad El Bosque. Bogotá DC, Colombia.

15 Médico, Especialista en Cirugía General y Cirugía de Colon y recto, Hospital San José y Clínica Los Nogales. Bogotá DC, Colombia.

16 Médico, Especialista en Cirugía General y Cirugía hepatobiliar, Servicio de trasplante hepático, Fundación Clínica Shaio. Bogotá DC, Colombia.

Palabras clave: COVID-I9; virus del SRAS; coronavirus; infecciones por coronavirus; pandemias; cirugía general; neoplasias. Key words: COVID-I9; SARS virus; coronavirus; coronavirus infections; pandemics; general surgery; neoplasms.

Fecha de recibido: 03/04/2020 - Fecha de aceptación: 05/04/2020

Correspondencia: Maikel A. Pacheco. Correo electrónico: maikel70@hotmail.com

Citar como: Pacheco MA, Torres PA, Arias F, Pinilla R, Abadía M, Villarreal R. Recomendaciones para cirugía en pacientes con patologías oncológicas durante la pandemia COVID-19. Rev Colomb Cir. 2020;35:162-70/Especial COVID-19.

https://doi.org/10.30944/20117582.616

Este es un artículo de acceso abierto bajo una Licencia Creative Commons - BY-NC-ND https://creativecommons.org/licenses/by-ncnd/4.0/deed.es 


\section{Generalidades}

Para emitir las recomendaciones específicas frente a la población de pacientes con cáncer, se tomaron en cuenta diversas observaciones en pacientes con patologías oncológicas en momentos de COVID-I9, que muestran riesgo mayor de complicaciones en esta población, así como las recomendaciones publicadas por las siguientes instituciones y asociaciones: National Comprehensive Cancer Network (NCCN), American College of Surgeons (ACS), American Society of Clinical Oncology (ASCO), European Society of Surgical Oncology (ESSO), Society of Surgical Oncology (SSO) y Asociación Española de Cirujanos (AEC).

$\mathrm{Si}$ bien las recomendaciones sobre cuidado personal y prevención de COVID-I9 para los pacientes con cáncer no difieren de las recomendaciones en la población general, es importante al momento de tomar decisiones relacionadas con su tratamiento, tener en cuenta todos los factores propios del entorno de cada paciente e institución, evaluando la situación epidemiológica de la zona, los recursos y talento humano disponibes para la atención, la condición particular y el entorno que rodea a cada paciente, con el fin de tomar la mejor decisión centrada en parámetros de bienestar y potencial control de la enfermedad.

La incorporación de estas recomendaciones a la práctica de cada grupo, debe seguir los criterios de buen juicio clínico y ético, proporcionalidad, prudencia y balance de las situaciones propias de cada paciente particular.

La atención de pacientes oncológicos se va modificando a medida que la crisis progresa en cada región, lo que se refleja en modificaciones sustanciales de la actividad asistencial en las áreas ambulatorias. El American College of Surgeons ha planteado tres fases que reflejan la progresión de la situación local de COVID-I9, que se consideran de gran utilidad para la toma de decisiones en cáncer:

Fase I: Entorno semi-urgente (fase de preparación): Pocos pacientes con COVID-I9, la institución todavía tiene disponibilidad para ventilación en la UCI y los casos COVID-I9 no se encuentran en fase de escalada rápida.
Recursos hospitalarios disponibles.

Fase II: Entorno urgente: Numerosos pacientes con COVID-I9, capacidad limitada de UCI y ventiladores, suministros para quirófano limitados.

Fase III: Todos los recursos hospitalarios se destinan a pacientes con COVID-I9, sin capacidad de ventiladores ni UCI. Quirófanos no disponibles excepto para situaciones de urgencia extrema. Suministros agotados.

Las recomendaciones expresadas a continuación, operan para las primeras fases de la crisis, en las cuales se reduce la atención en consulta y cirugía a casos considerados "prioritarios". Posteriormente en las fases siguientes, se suspende como consencuencia de una dedicación completa de la institución a los casos urgentes que trae la pandemia.

En las primeras fases de la pandemia, la realización de un triage y la selección de medidas teniendo en cuenta criterios de severidad y urgencia oncológica, permite la optimización de recursos a nivel institucional y del sistema de salud. Es ideal que la toma de decisiones esté apoyada por juntas multidisciplinarias que se pueden mantener en forma virtual con el fin de ofrecer a los pacientes acceso e información a las diferentes decisiones tomadas.

La "Escala de agudeza de cirugía electiva" (Elective Surgey Acuity Scale) propuesta por el doctor Samer Siddiqui de la Universidad de Saint Louis para establecer los criterios del triage oncológico y que fundamentó las recomendaciones del American College of Surgeons al respecto, puede ser de gran utilidad para nuestros servicios en la planeación de sus orientaciones generales para la toma de decisiones particulares en pacientes con cáncer.

El punto de partida supone un retraso de atención entre 3 a 6 meses, por lo que los riesgos de progresión tumoral con los retrasos en la programación de la cirugía definitiva deberán ser evaluados permanentemente ante la disponibilidad de recursos, la complejidad del paciente y el riesgo de exponerlo a adquirir COVID-ı en el medio hospitalario. 
Para casos electivos que tengan una alta probabilidad de requerir UCI y posible necesidad de ventilación mecánica, debe considerarse seriamente el riesgo-beneficio de retraso individual sobre la disponibilidad inminente de este tipo de recursos, para un paciente con COVID-I9 que los requiera, teniendo en cuenta que estas necesidades crecen día a día de forma exponencial.

\section{Recomendaciones generales:}

I. Los pacientes deben recibir atención quirúrgica adecuada y oportuna, basada en el buen juicio quirúrgico y la disponibilidad de recursos. Se deben evitar procedimientos quirúrgicos electivos en la noche, en estos horarios debe protegerse el equipo quirúrgico para momentos específicos de emergencia que probablemente sucedan.

2. Considerar el manejo no quirúrgico cuando sea clínicamente apropiado para el paciente y de acuerdo con las opciones disponibles para cada patología.

3. En pacientes potencialmente infectados se sugiere esperar los resultados de la prueba COVID-I9 para realizar el procedimiento quirúrgico. Sin embargo, dadas las dificultades de disponibilidad y tiempo para obtener los resultados en muchas regiones, se considera como una opción adecuada la realización de tomografía computarizada (TAC) de tórax preoperatorio para diagnóstico imaginológico.

4. Sólo en casos oncológicos que requieren cirugía urgente, en los que además no se cuente con las opciones mencionadas para descartar COVID-I9, se deben utilizar las máximas medidas de protección orientadas a la prevención del potencial contagio del grupo quirúrgico.

5. No se debe realizar cirugía por enfermedad benigna ni procedimientos quirúrgicos reductores de riesgo o profilácticos.

6. Con respecto a los pacientes ambulatorios, las entidades deberían tener comunicación con ellos y preguntar por síntomas relacionados con COVID-I9 para reagendar algunas consultas, así como las de los pacientes mayores de 70 años quienes serán atendidos una vez pase la etapa crítica de la pandemia o se catalogue su necesidad de atención como prioritaria.

7. Las consultas de primera vez por diagnóstico oncológico se considerarán una prioridad.

8. Se deberá informar a los pacientes que asistan a consulta externa, que sólo una persona deberá acompañarlos a sus consultas.

9. Se minimizará la cantidad de pacientes que asisten a las instituciones hospitalarias, sobre todo para actividades de seguimiento, donde las opciones de telemedicina serán recomendables.

\section{Recomendaciones frente a patologías específicas:}

\section{Cancer colorectal}

Las recomendaciones para la toma de decisiones en cáncer colorrectal se clasifican según las tres fases de la pandemia:

\section{Fase I:}

Incluyen casos que requieran intervención quirúrgica tan pronto como sea posible, considerando que la situación institucional se tornará cada vez más compleja durante las siguientes dos semanas. Estos pacientes son:

a) Aquellos con cáncer colorrectal con inminencia de obstrucción;

b) Pacientes con cáncer quienes requieren transfusiones frecuentes;

c) Pacientes con cáncer de colon sintomáticos;

d) Pacientes con cáncer de recto que no respondieron a la quimiorradiación neoadyuvante;

e) Tumores malignos con riesgo de perforación local y sepsis;

f) Aquellos con etapa temprana y, 
g) Pacientes con cáncer de recto que no son candidatos para la terapia adyuvante.

Fase II:

Comprende pacientes que necesitan cirugía tan pronto como sea posible, considerando la situación de la institución y probabilidad de requerimientos y limitación de recursos vitales para pacientes con COVID-I9. Estos casos incluyen pacientes con:

a) Cáncer de colon con obstrucción, donde la colocación de stent no es viable.

b) Cáncer de recto con inminencia de obstrucción (considerarse derivación endoscópica con stent o quirúrgica con ostomia).

c) Cáncer con alto requerimiento de transfusión (hospitalización); y

d) Cáncer con evidencia de perforación local y sepsis.

Todos los procedimientos colorrectales típicamente programados como rutina deben retrasarse y reagendarse al superar la pandemia.

\section{Fase III:}

Si es probable que el estado de la institución y la pandemia se vea más comprometido en las siguientes horas, la única cirugía que debe realizarse es para cánceres perforados, obstruidos, con sangrado activo (dependientes de transfusiones de pacientes hospitalizados) o con sepsis. En todos los demás casos las cirugías deben ser diferidas.

Los tumores malignos tempranos de colon generalmente progresan de manera relativamente lenta, con tiempos promedio de progresión entre 34 y 44 meses. El tratamiento integral basado en la cirugía no cambiará, pero debido al impacto de la pandemia, la combinación y la secuencia de tratamiento integral deberá ajustarse.

Las recomendaciones generales para el manejo del cáncer colorectal en tiempos de pandemia son:

I. Mientras sea posible para la institución, deben aplazar las cirugías para todos los póli- pos con cáncer y enfermedades en estadíos tempranos.

2. Realizar cirugía si el paciente está obstruido (Considerar derivación endoscópica con stent o quirúrgica con ostomia), o si está sangrando.

3. Realizar cirugía con intención curativa si es cáncer de colon.

4. Considerar todas las opciones de tratamiento neoadyuvante en pacientes con cáncer de recto y también en algunos de colon localmente avanzado.

5. Puede retrasarse cirugía post neoadyuvancia I2 a I6 semanas.

6. Para cancer de recto localmente avanzado, coordinar con el servicio de oncología radioterápica una dosis de radioterapia y posponer el procedimiento quirúrgico.

7. Evitar anastomosis primarias en pacientes de riesgo (anastomosis ultrabajas, diabéticos, mayores de 60 años, radioterapia preoperatoria), tanto por el alto riesgo de complicación sobreañadida de infección por COVID-I9 para el paciente, como para minimizar cuadros sépticos secundarios que puedan requerir recursos necesarios en el sistema de salud.

\section{Cáncer de seno}

\section{Fase I:}

Se deben restringir las cirugías a los pacientes que probablemente comprometan su supervivencia si la cirugía no se realiza en los siguientes 3 meses.

\section{Fase II:}

La cirugía debe estar restringida a los pacientes que verían comprometida su vida en días si no se realiza el procedimiento quirúrgico (se incluye aquí el manejo de las complicaciones postoperatorias recientes o abscesos que fallan al manejo percutáneo) 
Mientras sea posible, al revisar casos específicos, diferir al menos por 3 meses las cirugías de pacientes con atipia, cirugías para reducir riesgo o profilácticas, cirugías reconstructivas y cirugías por enfermedades benignas.

\section{Carcinoma ductal in situ}

Diferir por 3 a 5 meses, tratar ER + DCIS con terapia endocrina, monitorear mensualmente por progresión, garantizar prioridad a estos pacientes para el momento de tener disponibles espacios quirúrgicos una vez superada la situación actual.

\section{ER+ cáncer de seno invasivo (estadío I-III)}

Iniciar tratamiento endocrino o quimioterapia neoadyuvante, siguiendo las recomendaciones idealmente de una junta multidisciplinaria.

\section{Cáncer de mama invasivo triple negativo / HER 2+}

Tratar con quimioterapia neoadyuvante para la enfermedad T2 + y/o Ni. Considerar la cirugía primaria como una urgencia si el paciente no puede someterse a quimioterapia o si el tumor es pequeño y la información de la cirugía podría generar cambios en los esquemas de manejo.

\section{Pacientes en etapa posterior a quimioterapia neoadyuvante}

Retrasar la cirugía posterior a la quimioterapia el mayor tiempo posible, teniendo en cuenta una ventana de 4 a 8 semanas, sobretodo en los pacientes en quienes la adyuvancia no es clara o no está indicada.

\section{Casos inusuales, emergencias quirúrgicas}

Pacientes con enfermedad progresiva en terapia sistémica, angiosarcomas y tumor phylodes maligno deberán considerarse para cirugía de urgencia y no retrasar los procedimientos.

\section{Cáncer torácico}

Las pautas de cirugía de cáncer torácico siguen las del cáncer de seno.

\section{Fase I:}

Debe limitarse a pacientes cuya supervivencia puede verse afectada si la cirugía no se realiza dentro de los próximos 3 meses. Éstas incluyen:

I. Casos con cáncer de pulmón sólido o predominantemente sólido (>50\%) o presunto cáncer de pulmón $(>2 \mathrm{~cm})$, ganglio clínico negativo

2. Cáncer de pulmón con ganglios positivos

3. Cáncer de terapia post-inducción

4. Tumores de la pared torácica que son potencialmente agresivos y no manejables por medios alternativos

5. Etapas para comenzar el tratamiento (mediastinoscopia, cirugia torácica video asistida de diagnóstico para diseminación pleural)

6. Tumores mediastínicos sintomáticos.

7. Pacientes inscritos en ensayos clínicos terapéuticos.

Fase II:

Permitiría la cirugía si la supervivencia se viera afectada por un retraso de unos días. Estos casos incluirían cáncer de esófago perforado sin sepsis, una infección asociada a un tumor y manejo de complicaciones quirúrgicas en un paciente hemodinámicamente estable.

Todos los procedimientos torácicos considerados rutinarios / electivos serían diferidos.

\section{Fase III:}

Restringe la cirugía a pacientes cuya supervivencia se verá comprometida si no se someten a cirugía en las próximas pocas horas. Este grupo incluiría cáncer de esófago perforado en un paciente séptico, un paciente con una vía aérea amenazada, sepsis asociada con el cáncer y manejo de complicaciones quirúrgicas en un paciente inestable (sangrado activo que requiere cirugía, dehiscencia de la vía aérea, fuga anastomótica con sepsis). 
Todos los demás casos deberían ser diferidos. En general pueden considerarse diferibles en todas las fases los siguientes procedimientos:

a. Cirugía de nódulos sólidos o cáncer de pulmón menores de $2 \mathrm{~cm}$.,

b. Lesiones con histologías indolentes,

c. Timomas,

d. Oligometástasis pulmonares (a menos que puedan cambiar la orientación terapéutica),

e. Resecciones traqueales (solo en histologías agresivas ),

f. Broncoscopias

\section{Cáncer de cabeza y cuello y tumores endocrinos}

La mayoría de cirugías endocrinas no complicadas podrán ser diferidas.

Las enfermedades y presentaciones que podrían considerarse como urgentes (dentro de 4 a 8 semanas en la actual pandemia ) incluyen:

\section{Tiroides}

- Cuando amenaza la vida, por morbilidad dada su invasión local o por biología agresiva con recurrencia y rápido crecimiento

- Enfermedad de Graves sintomática que no ha tenido respuesta a manejo médico

- Bocio con síntomas compresivos en aumento, sobretodo inminencia de obstrucción de la vía aérea

- Biopsias por sospecha de tumores anaplásicos o linfomas

\section{Paratiroides}

- Hiperparatiroidismo con hipercalcemia potencialmente mortal que no se puede controlar médicamente

\section{Suprarrenal}

- Cáncer suprarenal o fuerte sospecha del mismo
- Sindrome de Cushing que no puede controlarse con manejo médico

Por lo general, tumores adrenales funcionales que están asintomáticos o pueden controlarse médicamente y adenomas adrenales no funcionales podrán ser diferidos.

\section{Tumores neuroendocrinos (TNE)}

- TNE del intestino delgado sintomáticos (sangrado, obstrucción, isquemia, etc,)

- TNE pancreáticos sintomáticos y/o funcionales que no pueden ser controlados médicamente

- Lesiones con crecimiento rápido

Cirugías de citorreducción y metastasectomías, en este contexto deberán ser diferidas, sin embargo, se recomienda considerarlas individualmente.

\section{Cáncer gástrico y esofágico}

En general, tiempos de espera preoperatorio de 6 meses no afectan el tiempo de sobrevida total en los pacientes con cáncer gástrico temprano, mientras el tiempo de espera de seguridad en los pacientes con cáncer gástrico en estadíos II y III idealmente debe ser menor de 3 meses.

Las recomendaciones de manejo del cáncer gástrico y esofágico en tiempos de pandemia son:

I. Las lesiones cTia susceptibles de manejo endoscópico, deberán ser manejadas preferiblemente de forma endoscópica.

2. Los cánceres cTib deberán ser manejados quirúrgicamente.

3. Los tumores $\mathrm{cT} 2$ o superior, o aquellos con evidencia de ganglios positivos, deberán ser llevados a neoadyuvancia.

4. Los pacientes que terminan neoadyuvancia y toleraron el tratamiento sin efectos adversos mayores, con respuesta clínica y paraclínica adecuada, pueden continuar con la quimio- 
terapia y aplazar los procedimientos quirúrgicos, más aún en cánceres biológicamente menos agresivos como Tumores Estromales Gastrointestinales (GIST), a menos que estén sintomáticos o sangrando.

5. Si el paciente está obstruido, considerar derivación endoscópica con stent o quirúrgica.

6. Si el paciente está sangrando, considerar radioterapia, embolización selectiva y/o manejo paliativo.

\section{Cáncer hepato-bilio-pancreático}

Realizar cirugías para todos los pacientes con neoplasias hepato-bilio-pancreáticas agresivas como esté indicado, específicamente en:

I. Adenocarcinoma de páncreas, colangiocarcinoma, cáncer periampular, metástasis colorrectales al hígado.

2. Si los pacientes se encuentran en quimioterapia neoadyuvante y hay respuesta favorable y adecuada y tolerancia al tratamiento, se recomienda continuar en quimioterapia y aplazar el procedimiento.

3. Se recomienda el uso de ablación o radiocirugía estereotáxica en lugar de resección quirúrgica para metástasis hepáticas en los lugares donde sea posible.

4. Considerar la ablación o la embolización sobre la resección quirúrgica para Hepatocarcinoma.

5. Aplazar los procedimientos quirúrgicos para Tumores Neuroendocrinos Pancreáticos (PNET) asintomáticos, adenomas duodenales y ampulares, GIST y Tumores Intraductales Papilares Mucinosas (IPMN) de alto riesgo, a menos que se considere que la demora pudiese afectar la resecabilidad.

\section{Melanoma}

Retrasar la escisión local amplia de la enfermedad in situ durante 3 meses y, a medida que los recursos de la institución disminuyan, también las de las lesiones con márgenes negativos en la biopsia inicial. Se deben hacer esfuerzos para realizar procedimientos en un entorno ambulatorio y así cuidar los recursos de las salas de cirugía.

El tratamiento quirúrgico de los melanomas $\mathrm{T}_{3} / \mathrm{T}_{4}$ ( $>2 \mathrm{~mm}$ de espesor) debe tener prioridad sobre melanomas Ti/T2 ( $<2 \mathrm{~mm}$ de espesor). La excepción es cualquier melanoma que sea de una biopsia parcial o incompleta en la que sea evidente una lesión residual de gran tamaño, en este caso se recomienda la resección completa.

La biopsia de ganglio linfático centinela se realizará a pacientes con lesiones > Imm de espesor y, cuando los recursos se vuelvan escasos, deberán aplazarse durante 3 meses.

Manejar la enfermedad clínica en estadio III con tratamiento neoadyuvante. Si el paciente no es adecuado para terapia sistémica, no hay disponibilidad en el medio y si los recursos aún lo permiten, considerar la resección quirúrgica en un entorno ambulatorio.

Las resecciones de metástasis (Etapas III y IV) pueden ser diferidas a menos que el paciente este crítico o sintomático, o no se tenga respuesta al tratamiento sistémico, teniendo en cuenta los recursos quirúrgicos disponibles.

\section{Malignidad de la superficie peritoneal}

I. La realización de Citoreducción completa más quimioterapia hipertérmica intraperitoneal (CRC+HIPEC) puede requerir el consumo de recursos únicos y valiosos, de tal manera que deben tenerse consideraciones especiales y ojalá derivadas de juntas clínicas multidisciplinarias, para determinar si dada la situación actual de la pandemia, es conveniente y pertinente su realización en ese momento o debe diferirse.

2. Diferir CRC+HIPEC en neoplasias mucinosas apendiculares de bajo grado, excepto en casos especiales con circunstancias discutidas y aprobadas en juntas multidisciplinarias. 
3. Considerar quimioterapia sistémica para metástasis peritoneales de cáncer de apéndice de alto grado, cáncer colorrectal, mesotelioma de alto grado, cáncer de ovario, cáncer gástrico y tumores desmoplásicos de células pequeñas y redondas.

4. Si los pacientes están completando la quimioterapia neoadyuvante y están listos para la cirugía, considere continuar la quimioterapia si hay respuesta favorable y adecuada y tolerancia al tratamiento, y además retrasar el tratamiento quirúrgico:

* 4-6 semanas en pacientes con carcinomatosis de origen en apéndice, colorrectal, mesotelioma de alto grado o cáncer de ovario.

* 2-4 semanas en pacientes con cáncer gástrico o tumores desmoplásicos de células pequeñas y redondas.

5. Aplazar la cirugía hasta superada la pandemia para metástasis peritoneales de tumores malignos raros de bajo grado como tumores neuroendocrinos, y tumores del estroma gastrointestinal.

6. Pacientes con obstrucción intestinal maligna, considerar llevarlos a cirugía de forma temprana para intentar un procedimiento paliativo oportunamente.

\section{Sarcoma}

- Dar prioridad a un sarcoma primario de tejidos blandos sin enfermedad metastásica.

- Diferir el tratamiento quirúrgico de los nuevos diagnósticos de liposarcomas bien diferenciados de tronco y extremidades, al igual que los tumores desmoides, por al menos 3 meses, tratando de garantizar el seguimiento y detectar por crecimiento o razones diferentes la necesidad de realizar la cirugía de forma prioritaria
- El manejo quirúrgico de otras lesiones de bajo grado con comportamientos más indolentes conocidos (por ejemplo, liposarcomas retroperitoneales bien diferenciados) y de bajo riesgo metastásico (por ejemplo, liposarcoma mixoide, tumor fibromixoide de bajo grado) pueden ser diferidos por intervalos cortos, dependiendo de los recursos disponibles

- Considerar diferir a intervalos cortos o ampliación de márgenes en procedimientos $\mathrm{RI}_{\mathrm{I}}$ en lesiones de tronco y extremidades, cuando los recursos sean limitados

- Considerar las opciones de radioterapia preoperatoria, que podría realizarse de forma ambulatoria y aplazaría el momento de la cirugía 3 a 4 meses

- La utilización de tratamiento neoadyuvante para sarcomas de alto grado o enfermedad recurrente puede ser considerado si existe un entorno ambulatorio seguro. Considerar también protocolos de observación activa $u$ opciones sistémicas de baja toxicidad para pacientes con enfermedad recurrente.

- La cirugía en pacientes con enfermedad recurrente podrá ofrecerse, siempre y cuando tengan posibilidades relativamente altas de obtener el control de la enfermedad a largo plazo en el contexto de resección macroscópica completa que requieran paliación inmediata, o los que tienen comportamiento biológico agresivo que no pueda manejarse con observación activa.

\section{Cumplimiento de normas éticas}

Elaborado y avalado por: Grupo de trabajo "Cirugía-ACC-Covid-ı9" de la Asociación Colombiana de Cirugía.

Consentimiento informado: Esta publicación es una revisión de la literatura, y como tal no hay necesidad de un consentimiento informado ni de aprobación del Comité de Ética Institucional.

Conflicto de intereses: Ninguno declarado por los autores. 


\section{Referencias}

American College of Surgeons. COVID-I9 Guidelines for triage of cancer surgery patients. Online March 24, 2020. Disponible en: https://www.facs.org/covid-I9/ clinical-guidance/elective-case/cancer-surgery

American society of clinical oncology. COVID-I9 Patient care information. Last updated on 04/02/2020. Disponible en: https://www.asco.org/asco-coronavirus-information/care-individuals-cancer-during-covid-I9

Asociación española de cirujanos. Documentos de posicionamiento y recomendaciones de la AEC en relación con la cirugía y COVID-I9. Disponible en: https:// www.aecirujanos.es/Documentos-de-posicionamiento-y-recomendaciones-de-la-AEC-en-relacion-con-la-cirugia-y-COVIDI9_es_I_I52.html

Bartlett D, Howe J. Cancer surgeries in the time of COVID-I9: A message from the SSO president and president elect. March 23, 2020. Disponible en: https:// www.surgonc.org/wp-content/uploads/2020/03/ COVID-I9-Letter-to-Members.pdf

Brindle M, Gawande A. Managing COVID-I9 in Surgical Systems. Annals of Surgery [Accepted for Publication]. 2020. Disponible en: https://journals.lww.
com/annalsofsurgery/Documents/Managing\%20 COVID\%2oin\%2oSurgical\%2oSystems\%20v2.pdf

Kovacs T. Advice for surgical oncologist on cancer service provision. European Society of Surgical Oncology (ESSO). Disponible en: https://essoweb.org/news/esso-statement-covid-I9/

Liang W, Guan W, Chen R, Wang W, Li J, Xu K, et al. Cancer patients in SARS-CoV-2 infection: a nationwide analysis in China. Lancet Oncol. 2020;2I:335-7. DOI: IO.IOI6/ Si470-2045(20)30096-6

Ma FH, Hu HT, Tian YT. Surgical treatment strategy for digestive system malignancies during the outbreak of novel coronavirus pneumonia. Chin J Oncol, 2020,42:Epub ahead of print. DOI: 10.3760/ cma.j.cnII2I52-20200223-00II7

Nelson R. Guidelines on delaying cancer surgery during COVID-I9. March 26, 2020. Disponible en: https:// www.medscape.com/viewarticle/927568

Ross SW. Maximizing the calm before the storm: a tiered surgical response plan for COVID-I9. J AM Coll Surg. March 2020. Disponible en: https://www.facs.org/-/ media/images/press-releases/2020/202O_cm_jacs_covidalert_pr_IIO4X736.ashx 